\title{
Integrierte Simulationssoftware für das Optimieren von Spritzgussvorgängen
}

Mit der Integration der Simulationssoftware Moldex3D in das Tool für digitale Produktentwicklung von Siemens PLM Software ermöglicht das Softwareunternehmen Simpatec das Optimieren von Spritzgussvorgängen. NX-Easy-Fill-Analysis dient der Simulation des Formfüllprozesses mit dem Ziel, die Konstruktion, Entwicklung und den Herstellprozess von Bauteilen für die Anwender von NX 8.5. stärker zu vereinfachen und zu beschleunigen.

Konstrukteure können nun Material- und Bauteileigenschaften direkt und genau analysieren und verifizieren. Dreidimensionale Ergebnisse aus der Analyse des Füll-, Nachdruck- oder Kühlverhaltens, Verzug, Faserorientierung, Einlegeteile etc. stehen zur
Verfügung, um das Produktdesign und die Herstellbarkeit zu optimieren, Markteinführungszeiten zu verkürzen und ROI-Kennziffern zu maximieren. Das Programm- und Analysetool ermöglicht es zudem, potenzielle Fertigungsfehler leicht zu überprüfen, ohne die gewohnte NX-Arbeitsumgebung zu verlassen. Es hilft den Anwendern signifikante Fragestellungen des Bauteildesigns effizienter zu beantworten, wie zum Beispiel bei der Auswahl der optimalen Zahl der Anspritzpunkte, des Materiales oder geeigneter Prozessbedingungen.

Mit Moldex3D eDesignSync für NX werden darüber erweiterte Funktionen und Aussagen hinsichtlich Nachdruckphase, Kühlung, Faserorientierung oder auch mög- liche Verzuge geboten. Es erlaubt laut Simpatec NX-Anwendern kritische Prozessfaktoren zu visualisieren und anhand von Ergebnissen, wie Einfallstellen, zu hohen Drücken oder thermischer Verzuge und das Schwindungsverhalten, optimale Herstellungsbedingungen zu finden.

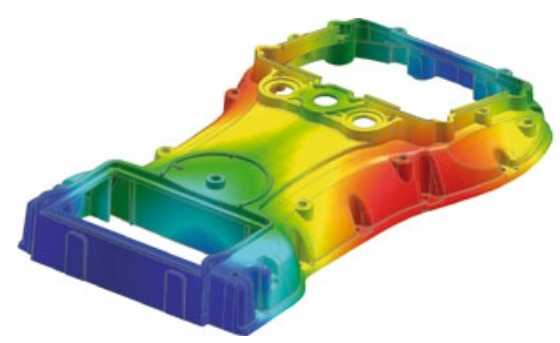

\section{Drehzahlvariable Antriebe im Pressenbau}

Vor etwas mehr als einem Jahr hat Langzauner mit seiner Laborpresse die drehzahlvariablen Antriebe in den Pressenbau eingeführt. Aufgrund der extremen Genauigkeit und Vielseitigkeit sind sie nach Angaben des Unternehmens für den Einsatz in Laborpressen prädestiniert. „Diese Pressen sind darauf ausgelegt, sämtliche Herstellungsprozesse zu beherrschen, und damit den Grundstein

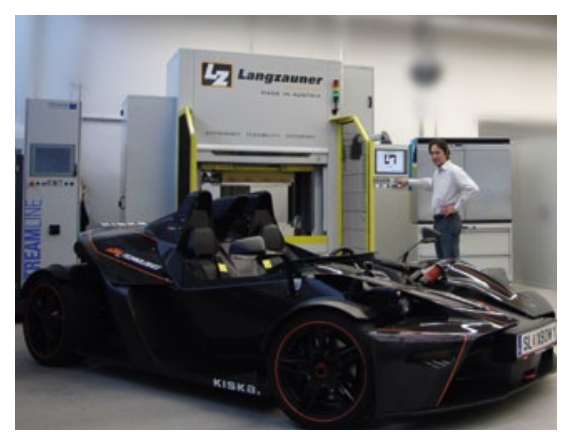

für eine effiziente Serienproduktion zu legen", heißt es. Dieses Konzept habe mittlerweile viele Forschungs- und Entwicklungsunternehmen überzeugt und sei mittlerweile mehrfach umgesetzt worden.

Eines der Hauptargumente dieses Systems sei aber seine überragende Energieeffizienz was auch für den Einsatz in Produktionspressen spreche. Durch die Vermeidung von Proportionalventilen ergäben sich alleine durch die mögliche Verwendung minderqualitativer Öle enorme Einsparungspotenziale. Das Öl sei darüber hinaus nur in Bewegung, wenn die Anlage eine Aktion ausführt. Dadurch erhöhe sich die Lebensdauer und der Einsatz eines Ölkühlers werde überflüssig. Die ersten Produktionspressen wurden bereits mit diesem System ausgerüstet, teilt Langzauner weiter mit. Die Einsparungspotenziale seien enorm, so habe sich bei einer 650-t-Presse im Dreischicht-Betrieb eine jähr- liche Einsparung im hohen fünfstelligen Bereich ergeben. Somit sei dieses Konzept vor allem für Zulieferer im Automobilbau sehr interessant, da aufgrund der hohen Stückzahlen hier die meisten Anstrengungen zum Thema Kosteneinsparung getätigt würden. Die Produkte zeichnen sich durch folgende Eigenschaften aus:

- geräuscharm

- hohe Genauigkeit

- energieeffizien

- Verwendung von Standard- Schaltventilen

- keine hohen Anforderungen an die Ölqualität

- längere Wartungsintervalle

- keine Einstell- und Justierarbeiten an Proportionalventilen

- keine Überprüfungspflichtigen Speicher

- keine Ölkühlung erforderlich

- Einsparungspotenzial Wartung, Öl tausch und Energie

- kein Überschwingen des Drucks. 